\title{
ANALYTICAL RESULTS ON A MODEL FOR DAMAGING IN DOMAINS AND INTERFACES*
}

\author{
Elena Bonetti ${ }^{1}$ And Michel Frémond ${ }^{2}$
}

\begin{abstract}
This paper deals with a model describing damage processes in a (nonlinear) elastic body which is in contact with adhesion with a rigid support. On the basis of phase transitions theory, we detail the derivation of the model written in terms of a PDE system, combined with suitable initial and boundary conditions. Some internal constraints on the variables are introduced in the equations and on the boundary, to get physical consistency. We prove the existence of global in time solutions (to a suitable variational formulation) of the related Cauchy problem by means of a Schauder fixed point argument, combined with monotonicity and compactness tools. We also perform an asymptotic analysis of the solutions as the interfacial damage energy (between the body and the contact surface) goes to $+\infty$.
\end{abstract}

Mathematics Subject Classification. 35K55, 74A15, 74M15.

Received June 24, 2009. Revised February 2, 2010.

Published online August 18, 2010.

\section{INTRODUCTION}

The investigation of damage in elastic materials is deeply studied in the literature both from analytical and theoretical point of view, as well as towards engineering applications. From a macroscopic description the process of damaging can be described as a lack of rigidity of the material. In the approach proposed by Frémond (see [13,16]) volume damage is described as the macroscopic effect of microscopic actions degenerating the cohesion of the material. A volume damage parameter $\beta$ is introduced to characterize the state of microbonds responsible for this cohesion. Then, the rigidity of the material (and thus the stress-strain relation) depends on $\beta$ and degenerates once the material is completely damaged (i.e. $\beta=0$ ). From an analytical point of view the problem is written by use of the theory of phase transitions and it has been studied both for elastic and viscoelastic materials, in the case of reversible and irreversible phenomena. We first recall some results holding in the one-dimensional setting (cf., e.g., [17]). Actually, the existence of a solution is proved during a finite time interval, i.e. till the material is completely damaged. Hence, some more recent papers deal with the more complicate situation of a $3 \mathrm{D}$ setting. The existence of a local in time solution is proved both

Keywords and phrases. Damage, contact, adhesion, existence, asymptotic analysis.

* This work has been supported by CMLA ENS-Cachan.

${ }^{1}$ Dipartimento di Matematica - Laboratoire Lagrange, Università di Pavia, via Ferrata 1, 27100 Pavia, Italy.

elena.bonetti@unipv.it

2 Centre de Mathématiques et de leurs Applications - Laboratoire Lagrange, École Normale Supérieure de Cachan, France.

fremond@cmla.ens-cachan.fr 
for (degenerating) elastic and viscoelastic laws (see $[4,5]$ ). The main problem consists in the degeneracy of the rigidity matrix: once the material is damaged deformations are not controlled. Thus, the quadratic source of damage involving deformations is not controlled, too. The possibility of finding a global in time existence result for the complete model is still an open problem. However, it seems that it cannot be overcome just by some more refined technical tools, as it is, in some sense, a mechanical weakness of the model itself which does not provide a suitable description of the material behaviour once it is completely damaged. In a recent paper [15] the authors, relying on soil mechanics properties, introduce a viscosity upgrading in the stress, forcing the deformation velocity to be bounded. The idea to add some non-degenerating viscosity can be found also in [2], combining as a novelty mechanical and thermal actions in the damaging.

In a similar framework, the theory of damage has been used to investigate the phenomenon of contact with adhesion between solids (see $[12,14,20]$ ). Indeed, in the classical unilateral theory for contact no resistance to tension was accounted for. In the case of adhesive contact the resistance to tension on the contact surface is due to microbonds between the surface of the body and its support. The state of such bonds describes the state of the adhesion, i.e. the adhesion is active if these bonds are not damaged, while there is no adhesion when the bonds get damaged. Thus, a surface damage parameter $\chi$ is introduced, for instance the surface fractions of micro-bonds, to describe the state of the adhesion. Hence, unilateral condition, i.e. impenetrability between solids, is ensured as an internal constraint on the boundary. The analytical formulation of the model has been recently introduced for reversible [6] and irreversible situations [7]. In a more recent paper thermal effects have been included in the model, influencing the phenomenon of the adhesion on the boundary [8].

The novelty of the present contribution consists in combining the theory of damage in domains with the phenomenon of the adhesion (see [10] for numerical results). More precisely, we investigate the behaviour of a body, located in a smooth bounded domain $\Omega \subseteq \mathbf{R}^{3}$, which can be damaged and which is in contact with adhesion on a rigid support on a part of its boundary $\Gamma_{c}$. The choice of dealing with a rigid support, instead of an elastic body that could be deformed and damaged, too, is just for the sake of simplicity. However, our modelling and analytical arguments could be extended, with some further technical difficulties, to describe the adhesion between two deformable solids. We consider the boundary $\partial \Omega=\Gamma_{1} \cup \Gamma_{2} \cup \Gamma_{c}$, where $\Gamma_{1}$ and $\Gamma_{c}$ have strictly positive measures.

We do not enter the details of the model and refer to [10] where a description of the model is given as well as some numerical simulations. Here, we introduce and investigate the corresponding analytical formulation, which is given in terms of an initial and boundary value problem. At a first analysis we are dealing with isothermal phenomena.

The main idea is to combine thermomechanical laws holding in the $3 \mathrm{D}$ domain $\Omega$ and on the boundary $\Gamma_{c}$. We assume that the state variables of the system, in terms of which the mechanical equilibrium is defined, are volume and surface variables. More precisely, we fix as state variables in $\Omega$ small deformations $\nabla u$, a damage parameter $\beta \in[0,1]$, and its gradient $\nabla \beta$. Note that the displacement $u$ is considered as a scalar (to avoid further technicalities in the analysis). On the surface contact $\Gamma_{c}$ we introduce a damage parameter for the adhesion $\chi \in[0,1]$, the gradient (on the surface) $\nabla \chi$, and the traces of the displacements $u_{\Gamma_{c}}$ and of the volume damage $\beta_{\left.\right|_{\Gamma_{c}}}$. Indeed, the mechanical equilibrium on the contact surface clearly depends also on the effects of volume damage and displacements. Analogously, the free energy of the system is split into two contributions: a surface (contact) free energy $\Psi_{\Gamma_{c}}$ and a volume one $\Psi_{\Omega}$. They are defined by

$$
\Psi_{\Omega}(\nabla u, \beta, \nabla \beta)=\frac{1}{2} \beta|\nabla u|^{2}+\frac{1}{2}|\nabla \beta|^{2}+I_{[0,1]}(\beta)+w(1-\beta),
$$

where $w>0$ accounts for cohesion and the indicator function $I_{[0,1]}(\beta)$ forces $\beta$ to assume only physically admissible values, as it is $I(\beta)=0$ if $\beta \in[0,1]$, while it is equal to $+\infty$ otherwise.

Then, on $\Gamma_{c}$ it is defined

$$
\Psi_{\Gamma_{c}}\left(\chi, \nabla \chi, u_{\Gamma_{\Gamma_{c}}}, \beta_{\left.\right|_{\Gamma_{c}}}\right)=\left.\left.\frac{\mu}{2} \chi\right|_{\left.\right|_{\Gamma_{c}}}\right|^{2}+w_{c}(1-\chi)+\frac{1}{2}|\nabla \chi|^{2}+I_{[0,1]}(\chi)+I_{(-\infty, 0]}\left(u_{\left.\right|_{\Gamma_{c}}}\right)+\frac{\nu}{2}\left|\beta_{\left.\right|_{\Gamma_{c}}}-\chi\right|^{2},
$$


$w_{c}>0$ being a cohesive constant, $\mu, \nu>0$. Let us briefly comment on the choice of the contact free energy. The indicator function $I_{[0,1]}$ forces $\chi$ to assume values in $[0,1]$. then, the impenetrability condition is given in terms of the function $I_{-}=I_{(-\infty, 0)}$, as it forces $u_{\Gamma_{c}}$ to assume negative values, prescribing as positive orientation the outward normal vector to the boundary. Then, two terms account for interactions between the body and the contact surface. The first one, depending on the positive parameter $\mu$, is actually responsible for the adhesion effect if $\chi>0$ ( $\mu \chi$ can be understood as the surface mechanical rigidity). The second one, depending on $\nu$, accounts for interactions between surface and volume damage [10]. It can force $\beta_{\left.\right|_{\Gamma_{c}}}$ and $\chi$ to be "not too far" and accounts for local interactions between volume and surface damages, it may be thought as a discrete gradient.

We introduce dissipation by use of the pseudo-potential of dissipation ( $c f .[19])$, that is a convex, non-negative functional attaining its minimum 0 if there is no dissipation. Actually, we introduce two functionals $\Phi_{\Omega}$ and $\Phi_{\Gamma_{c}}$, defined for dissipative variables in $\Omega$ and in $\Gamma_{c}$, respectively. More precisely, we set

$$
\Phi_{\Omega}\left(\beta_{t}\right)=\frac{1}{2}\left|\beta_{t}\right|^{2}
$$

and

$$
\Phi_{\Gamma_{c}}\left(\chi_{t}\right)=\frac{1}{2}\left|\chi_{t}\right|^{2}
$$

Let us point out that we are not requiring any constraint on the sign of the time derivatives of the damage parameters, as we assume the damage phenomena to be reversible (this is in particular the case of polymers or liquid glue).

To recover the equations of the model we use a generalized form of the principle of virtual powers $(c f$. . [13]) in which works and (micro)motions responsible for the damage processing are included. Consequently, we recover two balance equations holding in $\Omega$ (the momentum balance and a motion equation for the evolution of $\beta$ ) and an equation written in $\Gamma_{c}$ (a motion equation for the evolution of $\chi$ ).

More precisely, virtual velocities are given in $\Omega$, say $v$ for a virtual macroscopic velocity and $\gamma$ for a virtual microscopic velocity, and in $\Gamma_{c}$, say $\gamma_{s}$ a virtual microscopic contact velocity. Then, the power of interior forces is defined as follows

$$
\mathcal{P}_{i}\left(v, \gamma, \gamma_{s}\right)=-\int_{\Omega} \sigma \cdot \nabla v-\int_{\Gamma_{c}} R v-\int_{\Omega} B \gamma-\int_{\Omega} \mathbf{H} \cdot \nabla \gamma-\int_{\Gamma_{c}} R_{B}\left(\gamma-\gamma_{s}\right)-\int_{\Gamma_{c}} B_{s} \gamma_{s}-\int_{\Gamma_{c}} \mathbf{H}_{s} \cdot \nabla \gamma_{s},
$$

where $\sigma$ is the stress, $B, \mathbf{H}, B_{s}, \mathbf{H}_{s}$ are new interior forces in $\Omega$ and $\Gamma_{c}, R$ is a reaction on the contact surface, $R_{B}$ is an interaction term between damage in $\Omega$ and on the contact surface. The power of exterior forces is

$$
\mathcal{P}_{e}(v)=\int_{\Omega} f v+\int_{\Gamma_{2}} g v
$$

$g$ being a traction and $f$ a distance force.

We assume a quasi-static situation as the engineering applications are in contact mechanics and in civil engineering [10]. The case where the inertia forces are not neglected may involve collisions, i.e., velocity discontinuities. A mechanical model is available with some mathematical results [3,14]. The principle of virtual power reads

$$
\mathcal{P}_{i}+\mathcal{P}_{e}=0
$$


Then, prescribing the following constitutive relations

$$
\begin{aligned}
\sigma & =\frac{\partial \Psi_{\Omega}}{\partial \nabla u} \\
R & =\frac{\partial \Psi_{\Gamma_{c}}}{u_{\left.\right|_{\Gamma_{c}}}} \\
B & =\frac{\partial \Psi_{\Omega}}{\partial \beta}+\frac{\partial \Phi_{\Omega}}{\partial \beta_{t}} \\
\mathbf{H} & =\frac{\partial \Psi_{\Omega}}{\partial \nabla \beta} \\
R_{B} & =\frac{\partial \Psi_{\Gamma_{c}}}{\partial\left(\beta_{\Gamma_{\Gamma_{c}}}-\chi\right)} \\
B_{s} & =\frac{\partial \Psi_{\Gamma_{c}}}{\partial \chi}+\frac{\partial \Phi_{\Gamma_{c}}}{\partial \chi_{t}} \\
\mathbf{H}_{s} & =\frac{\partial \Psi_{\Gamma_{c}}}{\partial \nabla \chi},
\end{aligned}
$$

the equations are written as follows. We first have the momentum balance

$$
-\operatorname{div}(\beta \nabla u)=f \quad \text { in } \Omega
$$

where $\sigma=\beta \nabla u$, with boundary conditions

$$
\begin{aligned}
& u=0 \quad \text { on } \Gamma_{1}, \\
& (\beta \nabla u) \cdot \mathbf{n}=g \quad \text { on } \Gamma_{2}, \\
& (\beta \nabla u) \cdot \mathbf{n}=-R \in-\left(\mu \chi u_{\left.\right|_{\Gamma_{c}}}+\partial I_{-}\left(u_{\left.\right|_{\Gamma_{c}}}\right)\right) \quad \text { on } \Gamma_{c},
\end{aligned}
$$

$\mathbf{n}$ being the outward normal vector to the boundary. Let us comment about the reaction $R$. In the case the adhesion is not active, i.e. $\chi=0$, we recover Signorini conditions ensuring impenetrability between the body and the support, as $\partial I_{-}$is defined for $u_{\Gamma_{\Gamma_{c}}} \leq 0$ and it is $\partial I_{-}\left(u_{\left.\right|_{\Gamma_{c}}}\right)=0$ if $u_{\left.\right|_{\Gamma_{c}}}<0$, while $\partial I_{-}(0)=[0,+\infty)$. In the case $\chi>0$ the adhesion is active and a tension appears without separation. Then, we introduce the equation for $\beta$, which is of the form

$$
B-\operatorname{div} \mathbf{H}=0 \quad \text { in } \Omega
$$

with

It follows

$$
\mathbf{H} \cdot \mathbf{n}=0 \text { on } \Gamma_{1} \cup \Gamma_{2}, \quad \mathbf{H} \cdot \mathbf{n}=-R_{B} \text { on } \Gamma_{c} .
$$

combined with the boundary condition

$$
\beta_{t}-\Delta \beta+\partial I_{[0,1]}(\beta) \ni w-\frac{1}{2}|\nabla u|^{2},
$$

$$
\partial_{n} \beta=-\nu\left(\beta_{\Gamma_{\Gamma_{c}}}-\chi\right) \quad \text { on } \Gamma_{c}, \quad \partial_{n} \beta=0 \quad \text { otherwise. }
$$

Finally, in $\Gamma_{c}$ we address the balance equation (now the differential operators are defined in $\Gamma_{c}$, which is assumed for the sake of simplicity a flat surface)

$$
B_{s}-\operatorname{div} \mathbf{H}_{s}=0 \text { in } \Gamma_{c}, \quad \mathbf{H}_{s} \cdot \mathbf{n}_{s}=0 \text { on } \partial \Gamma_{c},
$$

$\mathbf{n}_{s}$ being the outward normal vector to the boundary of $\Gamma_{c}$. We get

$$
\chi_{t}-\Delta \chi+\partial I_{[0,1]}(\chi) \ni w_{c}-\frac{\mu}{2}\left|u_{\left.\right|_{\Gamma_{c}}}\right|^{2}+\nu\left(\beta_{\left.\right|_{\Gamma_{c}}}-\chi\right) .
$$


with

$$
\partial_{n} \chi=0
$$

on $\partial \Gamma_{c}$. Finally, initial conditions are prescribed for $\beta, \chi$

$$
\beta(0)=\beta_{0}, \quad \chi(0)=\chi_{0} .
$$

Note that, under suitable regularity on $\beta_{0}, \chi_{0}, f$, and $g$, we can get $u(0)=u_{0}$ defined as a solution of the corresponding equation (1.14) with (1.15).

In this paper, we are mainly interested to find a solution to the above equations (1.14) (actually we will deal with a slightly modified equation, see (1.24)), (1.18), and (1.21) combined with (1.15), (1.19), (1.22) and (1.23). We can prove a global in time existence result in any finite interval $(0, T), T>0$, for a weaker version of the problem. Uniqueness is really not expected due to the doubly nonlinear character of the system. The main difficulties we encounter are related to highly nonlinear terms in the equations and in the nonlinear coupling of them. First, we have to face with multivoque nonlinear operators, defined both in $\Omega$ and in $\Gamma_{c}$. In particular, let us point out that a nonlinear constraint is written in the boundary condition (1.15). Secondly, the quadratic source of the damage on the right hand side of (1.18) is not controlled once the material is damaged, due to the degeneracy of (1.24) once $\beta=0$. The analogous problem is related to the bound of the quadratic term involving the trace of displacement in (1.21) if $\chi=0$. To overcome this degeneracy of the model, we actually add a further nonlinearity $\frac{1}{4}|\nabla u|^{4}$ in $\Psi_{\Omega}$. The choice is physically justified by the theory of nonlinear elasticity: the stress in the powder or granular material where $\beta=0$ is very small if the strain is small and very large if the strain is large (a crude model of granular material). As already remarked, the predictive theory needs to be upgraded by new physical informations on the behaviour of the completely damaged material. Besides our choice, a large number of choices are possible depending on physics, see for instance [15]. Thus, the stress turns out to be defined by

and the equation for $u(c f .(1.14))$ turns out to be

$$
\sigma=|\nabla u|^{2} \nabla u+\beta \nabla u
$$

$$
-\operatorname{div}\left(|\nabla u|^{2} \nabla u+\beta \nabla u\right)=f .
$$

On a second step, we investigate the behaviour of our system once the interaction free energy goes to $+\infty$. More precisely, we let $\nu \rightarrow+\infty$ in the equations. At the limit we get that the trace of the volume damage $\beta_{\Gamma_{c}}$ and the surface damage parameter are forced to be the same. We are able to pass to the limit in (1.24), while we have to deal with (1.18) and (1.21) written as variational inequalities in a weaker framework. Indeed, we cannot control nonlinear constraints represented by maximal monotone operators independently of $\nu$. However, we get an interesting result as, at the limit, we get an evolution inequality for $\beta$ combined with the so-called "dynamic" boundary conditions. Let us point out that, in spite of the use of the adjective "dynamic", actually $\beta_{t}$ results from dissipation and not from inertial.

Here is the outline of the paper. In Section 2 we introduce a weak version of the problem and state the main existence result (Thm. 2.1). Hence, the proof of theorem is given in Section 3 by use of a fixed point argument, combined with a priori estimates and passage to the limit techniques. Finally, in Section 4 we perform the asymptotic analysis as $\nu \rightarrow+\infty$ (see Thm. 4.1).

\section{Mathematical Formulation AND MAin RESUlts}

In this section, we introduce the variational formulation of (1.24), (1.18), (1.21) combined with boundary assumptions (1.15), (1.19), (1.22). Then, we state the main existence result (see Thm. 2.1).

Before proceeding let us point out some useful notation and assumptions. Hereafter, for the sake of simplicity, we assume that $\Omega$ is a bounded smooth domain in $\mathbb{R}^{3}$, with $\partial \Omega=\bar{\Gamma}_{1} \cup \bar{\Gamma}_{2} \cup \overline{\Gamma_{c}}$, where $\Gamma_{1}, \Gamma_{2}$, and $\Gamma_{c}$ are open subsets in the relative topology of $\partial \Omega$, each of them with a smooth boundary and disjoint one from another; further, we assume that the contact surface $\Gamma_{c}$ and the region $\Gamma_{1}$ have strictly positive measure. Finally, for 
the sake of simplicity we let $\Gamma_{c} \subset \mathbb{R}^{2}$. Hence, given a Banach space $X$, we denote by ${ }_{X}{ }^{\prime}\langle\cdot, \cdot\rangle_{X}$ the duality pairing between $X^{\prime}$ and $X$; by the same symbol $\|\cdot\|_{X}$ we indicate both the norm in a Banach space $X$ and in any power of $X$. Finally, let $T>0$ be fixed. In the following, we may denote by the same symbol $c$ possibly different positive constants depending only on the data of the problem.

We introduce the Hilbert triplets

$$
V \hookrightarrow H \hookrightarrow V^{\prime}, \quad V_{c} \hookrightarrow H_{c} \hookrightarrow V_{c}^{\prime}
$$

with

$$
H:=L^{2}(\Omega), \quad V:=H^{1}(\Omega),
$$

and

$$
H_{c}:=L^{2}\left(\Gamma_{c}\right), \quad V_{c}:=H^{1}\left(\Gamma_{c}\right),
$$

where $H$ and $H_{c}$ are identified, as usual, with their dual spaces. Then, we set

$$
W:=\left\{v \in W^{1,4}(\Omega): v=0 \text { a.e. on } \Gamma_{1}\right\},
$$

endowed with the natural norm induced by $W^{1,4}(\Omega)$. Note in particular that, owing to the strictly positive measure of $\Gamma_{1}$, Poincaré's inequality leads to

$$
\|u\|_{W} \leq c(\Omega)\|\nabla v\|_{L^{4}(\Omega)} .
$$

We point out that if $v \in W$ then its trace belongs to $W^{3 / 4,4}(\Gamma)$. Then, let

$$
(-\infty, 0]_{W}=\left\{v \in W: v_{\left.\right|_{\Gamma_{c}}} \leq 0 \text { a.e. on } \Gamma_{c}\right\}
$$

and introduce the operator $\alpha=\partial_{W, W^{\prime}} I_{(-\infty, 0]_{W}}: W \rightarrow 2^{W^{\prime}}$, defined by

$$
\zeta \in \alpha(u) \text { if and only if } u \in(-\infty, 0]_{W}, W^{\prime}\langle\zeta, u-v\rangle_{W} \geq 0 \quad \forall v \in(-\infty, 0]_{W} .
$$

Analogously, we introduce $\gamma=\partial_{V, V^{\prime}} I_{[0,1]_{V}}: V \rightarrow V^{\prime}$ where

$$
[0,1]_{V}=\{\beta \in V: \beta \in[0,1] \text { a.e. in } \Omega\},
$$

defined by

$$
\xi \in \gamma(\beta) \text { if and only if } \beta \in[0,1]_{V}, V_{V^{\prime}}\langle\xi, \beta-\phi\rangle_{V} \geq 0 \quad \forall \phi \in[0,1]_{V} .
$$

Finally, we let $F \in W^{\prime}$ defined by

$$
W^{\prime}\langle F, v\rangle_{W}=\int_{\Omega} f v+\int_{\Gamma_{2}} g v, \quad v \in W .
$$

Before proceeding, let us fix the assumptions on the data of the problem. We first let

$$
F \in H^{1}\left(0, T ; W^{\prime}\right) .
$$

Then, we set

$$
\beta_{0} \in[0,1]_{V} ; \quad \chi_{0} \in V_{c}, \chi_{0} \in[0,1] \text { a.e. in } \Gamma_{c}
$$

and $\nu, \mu>0$.

Remark 2.1. Our existence results could apply to the simpler situation of $\mu=0$ (i.e. without adhesion between $\Omega$ and the contact surface) and $\nu=0$ (i.e., without any interaction between volume and surface damage). However, we prefer to explicitly consider the case of $\nu, \mu>0$ as it is more interesting both from analytical and modelling point of view. 
Here is the variational formulation of our problem.

The variational Problem $P_{v}$

Find $(u, \beta, \chi)$ such that for a.e. $t \in(0, T)$

$\int_{\Omega}|\nabla u|^{2} \nabla u \cdot \nabla v+\int_{\Omega} \beta \nabla u \cdot \nabla v+\mu \int_{\Gamma_{c}} \chi u_{\left.\right|_{\Gamma_{c}}} v_{\left.\right|_{\Gamma_{c}}}+{ }_{W^{\prime}}\langle\zeta, v\rangle_{W}=_{W^{\prime}}\langle F, v\rangle_{W} \quad \forall v \in(-\infty, 0]_{W}, \zeta \in \alpha(u)$ in $W^{\prime}$

$\int_{\Omega} \beta_{t} \phi+\int_{\Omega} \nabla \beta \cdot \nabla \phi+V^{\prime}\langle\xi, \phi\rangle_{V}+\nu \int_{\Gamma_{c}}\left(\beta_{\left.\right|_{\Gamma_{c}}}-\chi\right) \phi_{\left.\right|_{\Gamma_{c}}}=\int_{\Omega} w \phi-\frac{1}{2} \int_{\Omega}|\nabla u|^{2} \phi \quad \forall \phi \in[0,1]_{V}, \xi \in \gamma(\beta)$ in $V^{\prime}$,

$$
\chi_{t}-\Delta \chi+\delta=w_{c}-\frac{\mu}{2}\left|u_{\left.\right|_{\Gamma_{c}}}\right|^{2}-\nu\left(\chi-\beta_{\Gamma_{c}}\right), \delta \in \partial I_{[0,1]}(\chi) \quad \text { a.e. in } \Gamma_{c} .
$$

and fulfilling

$$
\beta(0)=\beta_{0}, \quad \chi(0)=\chi_{0} .
$$

The following theorem ensures existence of a solution to $P_{v}$.

Theorem 2.1. Let $T>0$ and assume that (2.4) and (2.5) hold. Then, there exists a solution $(u, \beta, \chi)$ to $P_{v}$ with the following regularity

$$
\begin{aligned}
& u \in L^{\infty}(0, T ; W), \\
& \beta \in H^{1}(0, T ; H) \cap L^{\infty}(0, T ; V), \\
& \chi \in H^{1}\left(0, T ; H_{c}\right) \cap L^{\infty}\left(0, T ; V_{c}\right) \cap L^{2}\left(0, T ; H^{2}\left(\Gamma_{c}\right)\right) .
\end{aligned}
$$

\section{A FIXED POINT ARGUMENT}

Our first step is to prove existence of a solution to Problem $P_{v}$ (see Thm. 2.1). To this aim, we apply the Schauder fixed point theorem to a slightly regularized version of $P_{v}$ in which a viscosity term is added in (3.1), i.e. we deal with the following equation for $\kappa>0$

$$
\begin{aligned}
\int_{\Omega} \beta_{t} \phi+\kappa \int_{\Omega} \nabla \beta_{t} \cdot \nabla \phi+\int_{\Omega} \nabla \beta \cdot \nabla \phi+V^{\prime}\langle\xi, \phi\rangle_{V}+\nu \int_{\Gamma_{c}}\left(\beta_{\left.\right|_{\Gamma_{c}}}-\chi\right) \phi_{\left.\right|_{\Gamma_{c}}} & =\int_{\Omega} w \phi-\frac{1}{2} \int_{\Omega}|\nabla u|^{2} \phi \\
& \forall \phi \in[0,1]_{V}, \quad \xi \in \gamma(\beta) \text { in } V^{\prime} .
\end{aligned}
$$

Remark 3.1. Let us point out that (3.1) describes a model for damage in which dissipative effects are included in the local interaction.

Let

$$
\begin{aligned}
& \mathcal{X}=\left\{(\phi, \psi) \in L^{\infty}(0, T ; H) \times L^{\infty}\left(0, T ; H_{c}\right)\right. \\
& \left.\phi, \psi \in[0,1] \text { a.e. in } \Omega \times(0, T) \text { and in } \Gamma_{c} \times(0, T) \text { respectively }\right\}
\end{aligned}
$$

$\mathcal{X}$ is endowed with the natural norm induced by $L^{\infty}(0, T ; H) \times L^{\infty}\left(0, T ; H_{c}\right)$. Hence, we are going to construct an operator

$$
\mathcal{T}: \mathcal{X} \rightarrow \mathcal{X}
$$

proving that it is compact and continuous (with respect to the topology of $\mathcal{X}$ ), whose fixed points define a solution to our problem. For the sake of simplicity, let us take $\mu=1$. 


\subsection{Auxiliary results}

First step. Let us fix

$$
(\widehat{\beta}, \widehat{\chi}) \in \mathcal{X} .
$$

We look at the solution $u=\mathcal{T}_{1}(\widehat{\beta}, \widehat{\chi})$ of the following abstract equation

$$
\mathcal{A} u+\mathcal{H}_{\widehat{\chi}} u+\operatorname{Div}(\widehat{\beta} \nabla u)+\zeta=F \quad \zeta \in \alpha(u), \quad \text { in } W^{\prime}, \text { a.e. in }(0, T),
$$

where $\alpha$ is introduced by (2.2) and the operators $\mathcal{A}, \mathcal{H}_{\hat{\chi}}: W \rightarrow W^{\prime}$ and Div $: H^{3} \rightarrow W^{\prime}$ are defined as follows. For $u, v \in W$

$$
\begin{aligned}
& W^{\prime}\langle\mathcal{A} u, v\rangle_{W}=\int_{\Omega}|\nabla u|^{2} \nabla u \cdot \nabla v, \\
& { }_{W^{\prime}}\langle\mathcal{H} \hat{\chi} u, v\rangle_{W}=\left.\int_{\Gamma_{c}} \widehat{\chi} u_{\left.\right|_{\Gamma_{c}}} v\right|_{\Gamma_{\Gamma_{c}}}, \\
& { }_{W^{\prime}}\langle\operatorname{Div}(\widehat{\beta} \nabla u), v\rangle_{W}=\int_{\Omega} \widehat{\beta} \nabla u \cdot \nabla v .
\end{aligned}
$$

The following lemma ensures the existence and uniqueness of a solution to (3.4).

Lemma 3.2. Let (2.4) hold. There exists a unique solution to (3.4). Moreover

$$
\|u\|_{L^{\infty}(0, T ; W)} \leq c_{1},
$$

for a constant $c_{1}>0$ depending on the data of the problem, but not on the choice of $(\widehat{\beta}, \widehat{\chi})$ in $\mathcal{X}$.

The existence of $u \in L^{\infty}(0, T ; W)$ solving (3.4) is proved exploiting well known-result on the theory of maximal monotone operators (for the general theory on maximal monotone operators in Banach spaces see, e.g., [1]). Concerning uniqueness, it follows by the fact that $\mathcal{A}$ satisfies (cf. [18])

$$
{ }_{W}\left\langle\mathcal{A}\left(u_{1}\right)-\mathcal{A}\left(u_{2}\right), u_{1}-u_{2}\right\rangle_{W}=0 \text { if and only if } u_{1}-u_{2}=0 .
$$

Indeed, as $\mathcal{A}, \mathcal{H}_{\hat{\chi}}$, Div (with fixed $\widehat{\beta}$ ), and $\alpha$ are monotone operators, if we write (3.4) for two solutions $u_{1}, u_{2}$, take the difference, and test by $u_{1}-u_{2}$, we get

$$
0 \leq_{W^{\prime}}\left\langle\mathcal{A}\left(u_{1}\right)-\mathcal{A}\left(u_{2}\right), u_{1}-u_{2}\right\rangle_{W} \leq 0,
$$

from which we deduce $u_{1}=u_{2}$ due to (3.9).

To prove (3.8) let us test (3.4) by $u$. We first observe that (see (2.2))

$$
W^{\prime}\langle\alpha(u), u\rangle_{W} \geq 0
$$

Hence, we have (independently of $t$ )

$$
\int_{\Omega}|\nabla u|^{4}+\int_{\Omega} \widehat{\beta}|\nabla u|^{2}+\int_{\Gamma_{c}} \widehat{\chi}|u|^{2} \leq_{W^{\prime}}\langle F, u\rangle_{W} .
$$

After exploiting the Young inequality and (2.1), it follows

$$
\|u\|_{W}^{4}+\int_{\Omega} \widehat{\beta}|\nabla u|^{2}+\int_{\Gamma_{c}} \widehat{\chi}|u|^{2} \leq c\|F\|_{W^{\prime}}^{4 / 3}
$$


from which (3.8) is deduced as $\widehat{\beta}, \widehat{\chi}>0$ and $F$ satisfies (2.4). In addition, we infer that

$$
\left\|\widehat{\chi}^{1 / 2} u_{\Gamma_{\Gamma_{c}}}\right\|_{L^{\infty}\left(0, T ; H_{c}\right)}+\left\|\widehat{\beta}^{1 / 2} \nabla u\right\|_{L^{\infty}(0, T ; H)} \leq c_{1} .
$$

Note that (3.8) yields

$$
\left\||\nabla u|^{2} \nabla u\right\|_{L^{\infty}\left(0, T ; L^{4 / 3}(\Omega)\right)} \leq c,
$$

from which, by a comparison in (3.4), the following bound is recovered

$$
\|\zeta\|_{L^{\infty}\left(0, T ; W^{\prime}\right)} \leq c
$$

Second step. Assume (2.5) and (2.4). Fix $u=\mathcal{T}_{1}(\widehat{\beta}, \widehat{\chi})$ (defined by Lem. 3.2) and $\widehat{\chi}$. Then, let us consider the equation in $V^{\prime}$ for a.e. $t \in(0, T)$

$$
\beta_{t}+\kappa A_{0} \beta_{t}+A \beta+\xi=w-\frac{1}{2}|\nabla u|^{2}+T_{\hat{\chi}}, \quad \xi \in \gamma(\beta)
$$

where

$$
\begin{aligned}
& A, A_{0}: V \rightarrow V^{\prime}, \quad V^{\prime}\langle A \beta, \phi\rangle_{V}=\int_{\Omega} \nabla \beta \cdot \nabla \phi+\nu \int_{\Gamma_{c}} \beta_{\Gamma_{\Gamma_{c}}} \phi_{\Gamma_{\Gamma_{c}}}, \\
& V^{\prime}\left\langle A_{0} \beta_{t}, \phi\right\rangle_{V}=\int_{\Omega} \nabla \beta_{t} \cdot \nabla \phi
\end{aligned}
$$

and

$$
{ }^{\prime}\left\langle T_{\hat{\chi}}, \phi\right\rangle_{V}=\nu \int_{\Gamma_{c}} \widehat{\chi}_{\phi_{\Gamma_{c}}}
$$

for any $\phi \in V$. The following lemma holds.

Lemma 3.3. There exists a unique solution $\beta=\mathcal{T}_{2}(u, \hat{\chi})$ to (3.16), (2.9). Moreover, it is

$$
\|\beta\|_{H^{1}(0, T ; V)} \leq c_{2},
$$

where $c_{2}$ is independent of $\widehat{\beta}$ and $\widehat{\chi}$.

The existence and uniqueness result stated by Lemma 3.3 is fairly standard and follows from the theory of evolution (parabolic) equations, associated with maximal monotone operators, whose right hand side is in $L^{\infty}(0, T ; H)+L^{\infty}\left(0, T ; V^{\prime}\right)$. Hence, let us proof the uniform bound (3.18). We test (3.1) by $\beta_{t}$ and integrate over $(0, t)$. We get, exploiting Young's inequality and trace theorems,

$$
\begin{aligned}
\left\|\beta_{t}\right\|_{L^{2}(0, t ; V)}^{2}+\frac{1}{2}\|\nabla \beta(t)\|_{H}^{2}+\frac{\nu}{2}\left\|\beta_{\Gamma_{\Gamma_{c}}}(t)\right\|_{H_{c}}^{2} \leq & \frac{1}{2}\left\|\nabla \beta_{0}\right\|_{H}^{2}+\int_{0}^{t} \int_{\Omega} w\left|\beta_{t}\right|+\frac{1}{2} \int_{0}^{t} \int_{\Omega}|\nabla u|^{2}\left|\beta_{t}\right| \\
& +\nu \int_{0}^{t} \int_{\Gamma_{c}} \widehat{\chi}\left|\beta_{t}\right|+\frac{\nu}{2}\left\|\beta_{\left.\right|_{\Gamma_{c}}}(0)\right\|_{H_{c}}^{2} \\
\leq & c\left(1+\int_{0}^{t}\|\nabla u\|_{L^{4}(\Omega)}^{4}+\|\widehat{\chi}\|_{L^{2}\left(0, T ; H_{c}\right)}^{2}\right)+\frac{1}{2}\left\|\beta_{t}\right\|_{L^{2}(0, t ; V)}^{2} \\
\leq & c_{2}+\frac{1}{2}\left\|\beta_{t}\right\|_{L^{2}(0, t ; V)}^{2},
\end{aligned}
$$


where $c_{2}$ depends on $c_{1}$ and on the data of the problem (see (3.8)), but not on the choice of $\widehat{\beta}$ and $\widehat{\chi}$. Note that we have exploited the fact that, by chain rule,

$$
\int_{0}^{t} V^{\prime}\left\langle\xi, \beta_{t}\right\rangle_{V} \geq 0
$$

Thus, (3.18) easily follows. Then, a comparison in (3.16) yields

$$
\|\xi\|_{L^{\infty}\left(0, T ; V^{\prime}\right)} \leq c_{2} .
$$

Third step. Let $u=\mathcal{T}_{1}(\widehat{\beta}, \widehat{\chi})$ and $\beta=\mathcal{T}_{2}(u, \widehat{\chi})$ defined by Lemmas 3.2 and 3.3, once (2.4) and (2.5) are assumed. Consider the following equation, written in $\Gamma_{c} \times(0, T)$

$$
\chi_{t}-\Delta \chi+\delta+\nu \chi=w_{c}-\frac{1}{2}\left|u_{\left.\right|_{\Gamma_{c}}}\right|^{2}+\nu \beta_{\left.\right|_{\Gamma_{c}}}, \quad \delta \in \partial I_{[0,1]}(\chi) .
$$

Lemma 3.4. There exists a unique solution $\chi=\mathcal{T}_{3}(u, \beta)$ to (3.21), (2.9) with

$$
\|\chi\|_{H^{1}\left(0, T ; H_{c}\right) \cap L^{\infty}\left(0, T ; V_{c}\right) \cap L^{2}\left(0, T ; H^{2}\left(\Gamma_{c}\right)\right)} \leq c_{3},
$$

where $c_{3}$ does not depend on $\widehat{\beta}$ and $\widehat{\chi}$ but only on the data of the problem.

We first observe that, by trace theorems and Sobolev's embedding, (3.18) implies

$$
\left\|\beta_{\left.\right|_{\Gamma_{c}}}\right\|_{L^{\infty}\left(0, T ; L^{4}\left(\Gamma_{c}\right)\right)} \leq c .
$$

Analogously, due to (3.8) we get, at least, for any $p$

$$
\left\|u_{\Gamma_{\Gamma_{c}}}\right\|_{L^{\infty}\left(0, T ; L^{p}\left(\Gamma_{c}\right)\right)} \leq c .
$$

Thus, the right hand side of (3.21) turns out to be bounded in $L^{\infty}\left(0, T ; L^{4}\left(\Gamma_{c}\right)\right)$ and existence and uniqueness of the solution to (3.16), (2.9) follows by standard arguments $(c f .[1,7])$. Then, to show that (3.22) holds, let us test the equation $(3.21)$ by $\chi_{t}$ and integrate over $(0, t)$. It is now a standard matter to infer that

$$
\left\|\chi_{t}\right\|_{L^{2}\left(0, t ; H_{c}\right)}^{2}+\|\nabla \chi(t)\|_{H_{c}}^{2}+\frac{\nu}{2}\|\chi(t)\|_{H_{c}}^{2} \leq c\left(1+\int_{0}^{t}\left\|u_{\Gamma_{\Gamma_{c}}}\right\|_{L^{4}\left(\Gamma_{c}\right)}^{4}+\nu \int_{0}^{t}\left\|\beta_{\left.\right|_{\Gamma_{c}}}\right\|_{H_{c}}^{2}\right) \leq c
$$

where $c$ depends on the data of the problem and, in particular, on $c_{1}$ and $c_{2}$. Hence, we can test (3.21) by $-\Delta \chi$. After integrating over $(0, t)$ and observing that by monotonicity

$$
\int_{\Omega} \nabla \delta \cdot \nabla \chi \geq 0
$$

it follows that

$$
\|\Delta \chi\|_{L^{2}\left(0, T ; H_{c}\right)}^{2} \leq c
$$

Combining (3.25) and (3.26) we get (3.22) and, by a comparison in (3.21)

$$
\|\delta\|_{L^{2}\left(0, T ; H_{c}\right)} \leq c .
$$




\subsection{Existence of the operator $\mathcal{T}$ and of a fixed point}

Combining $\mathcal{T}_{1}, \mathcal{T}_{2}$, and $\mathcal{T}_{3}$, due to Lemmas 3.2-3.4, we are in the position of well define an operator $\mathcal{T}: \mathcal{X} \rightarrow \mathcal{X}$ as follows

$$
\mathcal{T}(\widehat{\beta}, \widehat{\chi})=(\beta, \chi) \quad \text { where } \beta=\mathcal{T}_{2}(u, \widehat{\chi}), \quad u=\mathcal{T}_{1}(\widehat{\beta}, \widehat{\chi}), \quad \chi=\mathcal{T}_{3}(u, \beta) .
$$

Note in particular that, by construction, a fixed point $(\beta, \chi)$ of $\mathcal{T}$ provides a solution to $P_{v}$ (in which (3.1) is considered for $\kappa>0)(u, \beta, \chi)$ defined by (3.28).

Now, to apply the Schauder theorem, and deduce that $\mathcal{T}$ admits a fixed point, we need to show that it is compact and continuous in $\mathcal{X}$ w.r.t. the topology induced by $L^{\infty}(0, T ; H) \times L^{\infty}\left(0, T ; H_{c}\right)$.

As far as compactness, it easily follows from (3.18) and (3.22) (holding for constants depending only on the data of the problem), due to [21].

Then, it remains to prove that the operator $\mathcal{T}$ is continuous. To this aim let us take $\left(\widehat{\beta}_{n}, \widehat{\chi}_{n}\right) \in \mathcal{X}$ such that

$$
\begin{aligned}
& \widehat{\beta}_{n} \rightarrow \widehat{\beta} \quad \text { in } L^{\infty}(0, T ; H) \\
& \widehat{\chi}_{n} \rightarrow \widehat{\chi} \quad \text { in } L^{\infty}\left(0, T ; H_{c}\right) .
\end{aligned}
$$

Our aim is to show that

$$
\left(\beta_{n}, \chi_{n}\right)=\mathcal{T}\left(\widehat{\beta}_{n}, \widehat{\chi}_{n}\right) \rightarrow \mathcal{T}(\widehat{\beta}, \widehat{\chi}) \quad \text { in } L^{\infty}(0, T ; H) \times L^{\infty}\left(0, T ; H_{c}\right) .
$$

Let us denote by

$$
u_{n}=\mathcal{T}_{1}\left(\widehat{\beta}_{n}, \widehat{\chi}_{n}\right), \quad \beta_{n}=\mathcal{T}_{2}\left(u_{n}, \widehat{\chi}_{n}\right), \quad \chi_{n}=\mathcal{T}_{3}\left(u_{n}, \beta_{n}\right) .
$$

Then, by $\zeta_{n}, \xi_{n}, \delta_{n}$ we denote the selections of $\alpha, \gamma, \partial I_{[0,1]}$ in (3.4), (3.16), (3.21) written for the index $n$.

We first recall that (3.8), (3.18), and (3.22) imply

$$
\begin{aligned}
& \left\|u_{n}\right\|_{L^{\infty}(0, T ; W)} \leq c, \\
& \left\|\beta_{n}\right\|_{H^{1}(0, T ; V)} \leq c, \\
& \left\|\chi_{n}\right\|_{H^{1}\left(0, T ; H_{c}\right) \cap L^{\infty}\left(0, T ; V_{c}\right) \cap L^{2}\left(0, T ; H^{2}\left(\Gamma_{c}\right)\right)} \leq c,
\end{aligned}
$$

where $c$ does not depend on $n$. Thus, by weak star compactness results, at least for some suitable subsequences (still denoted by the index $n$ just for the sake for simplicity), we deduce (at least)

$$
\begin{aligned}
& u_{n} \stackrel{*}{*} u \quad \text { in } L^{\infty}(0, T ; W), u_{n} \rightarrow u \quad \text { in } L^{4}(0, T ; W), \\
& \beta_{n} \rightarrow \beta \quad \text { in } H^{1}(0, T ; V), \\
& \chi_{n} \stackrel{*}{\rightarrow} \quad \text { in } H^{1}\left(0, T ; H_{c}\right) \cap L^{\infty}\left(0, T ; V_{c}\right) \cap L^{2}\left(0, T ; H^{2}\left(\Gamma_{c}\right)\right) .
\end{aligned}
$$

Let us point out that to perform the following asymptotic analysis would be sufficient to have

$$
\left\|\beta_{n}\right\|_{H^{1}(0, T ; H) \cap L^{\infty}(0, T ; V)}, \quad \beta_{n} \stackrel{*}{\rightarrow} \beta \quad \text { in } H^{1}(0, T ; H) \cap L^{\infty}(0, T ; V) .
$$

Hence, strong compactness theorems ensure

$$
\begin{aligned}
& \beta_{n} \rightarrow \beta \quad \text { in } C^{0}\left([0, T] ; H^{1-\varepsilon}(\Omega)\right), \varepsilon>0, \\
& \chi_{n} \rightarrow \chi \quad \text { in } C^{0}\left([0, T] ; H^{1-\varepsilon}\left(\Gamma_{c}\right)\right) \cap L^{2}\left(0, T ; H^{2-\varepsilon}\left(\Gamma_{c}\right)\right), \varepsilon>0 .
\end{aligned}
$$

In particular, owing to Sobolev's embedding and trace theorems (3.40) imply

$$
\begin{aligned}
& \beta_{n} \rightarrow \beta \quad \text { in } C^{0}\left([0, T] ; L^{p}(\Omega)\right), p<6, \\
& \beta_{\left.n\right|_{\Gamma_{c}}} \rightarrow \beta_{\left.\right|_{\Gamma_{c}}} \text { in } C^{0}\left([0, T] ; L^{p}\left(\Gamma_{c}\right)\right), p<4 .
\end{aligned}
$$


Analogously, as far as the convergence of the trace of $u_{n},(3.33)$ and (3.36) yield

$$
u_{\left.n\right|_{\Gamma_{c}}} \stackrel{*}{\rightarrow} u_{\Gamma_{\Gamma_{c}}} \quad \text { in } L^{\infty}\left(0, T ; W^{3 / 4,4}\left(\Gamma_{c}\right)\right),
$$

and consequently a weak convergence holds, e.g., in $L^{p}\left(0, T ; L^{p}\left(\Gamma_{c}\right)\right)$, for $1<p<+\infty$. Then, (3.41) leads to

$$
\chi_{n} \rightarrow \chi \quad \text { in } C^{0}\left([0, T] ; L^{p}\left(\Gamma_{c}\right)\right), p<+\infty
$$

Now, let us comment about $\zeta_{n}, \xi_{n}, \delta_{n}$. We recall that (3.15), (3.20), and (3.27) imply

$$
\begin{gathered}
\left\|\zeta_{n}\right\|_{L^{\infty}\left(0, T ; W^{\prime}\right)} \leq c \\
\left\|\xi_{n}\right\|_{L^{2}\left(0, T ; V^{\prime}\right)} \leq c \\
\left\|\delta_{n}\right\|_{L^{2}\left(0, T ; H_{c}\right)} \leq c
\end{gathered}
$$

independently of $n$. Then, the following convergences follow

$$
\begin{aligned}
& \zeta_{n} \stackrel{*}{\rightarrow} \zeta \text { in } L^{\infty}\left(0, T ; W^{\prime}\right), \quad \zeta_{n} \rightarrow \zeta \text { in } L^{2}\left(0, T ; W^{\prime}\right) \\
& \xi_{n} \rightarrow \xi \quad \text { in } L^{2}\left(0, T ; V^{\prime}\right) \\
& \delta_{n} \rightarrow \delta \text { in } L^{2}\left(0, T ; H_{c}\right) .
\end{aligned}
$$

Now, let us deal with the passage to the limit in (3.4) as $n \rightarrow+\infty$. We first observe that $\eta_{n}=\left|\nabla u_{n}\right|^{2} \nabla u_{n}$ is bounded in $L^{\infty}\left(0, T ; L^{4 / 3}(\Omega)\right)$ (see $\left.(3.14)\right)$, so that

$$
\eta_{n}=\left|\nabla u_{n}\right|^{2} \nabla u_{n} \stackrel{*}{\rightarrow} \eta \quad \text { in } L^{\infty}\left(0, T ; L^{4 / 3}(\Omega)\right) .
$$

This implies that

$$
\begin{aligned}
& \left\|\mathcal{A}\left(u_{n}\right)\right\|_{L^{\infty}\left(0, T ; W^{\prime}\right)} \leq c, \\
& \mathcal{A} u_{n} \stackrel{*}{\rightarrow} E, \quad \text { in } L^{\infty}\left(0, T ; W^{\prime}\right), \quad \mathcal{A} u_{n} \rightarrow E \quad \text { in } L^{2}\left(0, T ; W^{\prime}\right),
\end{aligned}
$$

where

$$
W^{\prime}\langle E, v\rangle_{W}=\int_{\Omega} \eta \cdot \nabla v, \quad v \in W .
$$

Owing to (3.43) and (3.44) for any $v \in W$ as $n \rightarrow+\infty$ there holds

$$
\int_{\Gamma_{c}} \widehat{\chi}_{n} u_{\left.n\right|_{\Gamma_{c}}} v_{\left.\right|_{\Gamma_{c}}} \rightarrow \int_{\Gamma_{c}} \widehat{\chi} u_{\left.\right|_{\Gamma_{c}}} v_{\left.\right|_{\Gamma_{c}}} .
$$

Analogously, (3.36) and (3.37) imply

$$
\int_{\Omega} \widehat{\beta}_{n} \nabla u_{n} \cdot \nabla v \rightarrow \int_{\Omega} \widehat{\beta} \nabla u \cdot \nabla v .
$$

Eventually, exploiting (3.36), (3.48), (3.52) (see (3.51)), (3.54), and (3.55), we can pass to the limit weakly in $(3.4)$ and get the following weak equation in $W^{\prime}$

$$
E+\zeta+\mathcal{H}_{\widehat{\chi}} u+\operatorname{Div}(\widehat{\beta} \nabla u)=F
$$

In particular, we have to identify $E \in \mathcal{A} u$ and $\zeta \in \alpha(u)$. We apply an analogous argument as that exploited in Lemma 5.1 of [9] for the Hilbert case. 
Lemma 3.5. Let $X, X^{\prime}$ be reflexive Banach spaces and $F: X \rightarrow X^{\prime}$ a duality mapping, $B$ a maximal monotone set in $X \times X^{\prime}$. Suppose that $\left(x_{n}, y_{n}\right) \in B$ for any $n \in \mathbb{N}$ and for $n \rightarrow+\infty$

$$
x_{n} \rightarrow x \text { in } X, \quad y_{n} \rightarrow y \text { in } X^{\prime} .
$$

Then, denoting by $\langle\cdot, \cdot\rangle$ the duality pairing between $X^{\prime}$ and $X$,

$$
\liminf _{n \rightarrow+\infty}\left\langle y_{n}, x_{n}\right\rangle \geq\langle y, x\rangle
$$

Let us briefly comment about the proof of the above result. As $B$ is monotone, for any $(\omega, \rho) \in B$ there holds

$$
\left\langle y_{n}-\rho, x_{n}-\omega\right\rangle \geq 0 \quad \forall n
$$

Thus, passing to the limit we have

$$
\liminf _{n \rightarrow+\infty}\left\langle y_{n}, x_{n}\right\rangle \geq\langle y, \omega\rangle+\langle\rho, x\rangle-\langle\rho, \omega\rangle,
$$

for any $(\omega, \rho) \in B$. Now, let us take, for $\lambda>0$, the resolvent $J_{\lambda}$ and the Yosida approximation $B_{\lambda}$ of $B$ (cf. [1] p. 41). Let $\omega=J_{\lambda} x$ and $\rho=A_{\lambda} x$. By definition, we have

$$
A_{\lambda} x=-\lambda^{-1} F\left(J_{\lambda} x-x\right)
$$

Then, it follows

and due to $(3.57)$

$$
\langle\rho, x-\omega\rangle=\left\langle-\lambda^{-1} F\left(J_{\lambda} x-x\right), J_{\lambda} x-x\right\rangle \geq 0
$$

$$
\liminf _{n \rightarrow+\infty}\left\langle y_{n}, x_{n}\right\rangle \geq\left\langle y, J_{\lambda} x\right\rangle
$$

Now, due to [1], Corollary 1.2, the strong closure of $D(B)$ is convex. Thus, it coincides with the weak closure of $D(B)$. In particular, we get that $x$ belongs to the strong closure of $D(B)$, so that $J_{\lambda} x \rightarrow x$ as $\lambda \rightarrow 0$ due to [1], Proposition 1.1, which concludes the proof of the lemma passing to the limit in (3.58).

Applying Lemma 3.5 in the duality of $L^{2}(0, T ; W)$ with $L^{2}\left(0, T ; W^{\prime}\right)$, owing to $(3.36)$ and (3.48). It follows

$$
\liminf _{n \rightarrow+\infty} \int_{0}^{t} W^{\prime}\left\langle\zeta_{n}, u_{n}\right\rangle_{W} \geq \int_{0}^{t} W^{\prime}\langle\zeta, u\rangle_{W}
$$

Now, let us consider (3.4) written for $n$ (denoting by $E_{n}=\mathcal{A} u_{n}$ ). Test it by $u_{n}$ and integrate over $(0, t)$

$$
\begin{aligned}
\limsup _{n \rightarrow 0} \int_{0}^{t} W^{\prime}\left\langle E_{n}, u_{n}\right\rangle W \leq & -\liminf _{n \rightarrow+\infty} \int_{0}^{t} \int_{\Gamma_{c}} \widehat{\chi}_{n}\left|u_{n}\right|^{2}-\liminf _{n \rightarrow+\infty} \int_{0}^{t} \int_{\Omega} \widehat{\beta}_{n}\left|\nabla u_{n}\right|^{2} \\
& +\lim _{n \rightarrow+\infty} \int_{0}^{t} W^{\prime}\left\langle F, u_{n}\right\rangle_{W}-\liminf _{n \rightarrow+\infty} \int_{0}^{t} W^{\prime}\left\langle\zeta_{n}, u_{n}\right\rangle_{W} \\
\leq & -\int_{0}^{t} \int_{\Gamma_{c}} \widehat{\chi}|u|^{2}-\int_{0}^{t} \int_{\Omega} \widehat{\beta}|\nabla u|^{2}+\int_{0}^{t} W^{\prime}\langle F, u\rangle_{W}-\int_{0}^{t} W^{\prime}\langle\zeta, u\rangle_{W}
\end{aligned}
$$

Let us comment about (3.60). Due to (3.30), at least for some subsequence, $\widehat{\chi}_{n}^{1 / 2} \rightarrow \widehat{\chi}^{1 / 2}$ a.e. and strongly, e.g., in $L^{q}$ for $q<+\infty$. Thus, recalling that (3.43) holds and $\widehat{\chi}_{n}^{1 / 2} u_{n}$ is bounded in $L^{\infty}\left(0, T ; H_{c}\right)(c f$. (3.13)), we can pass to the limit

$$
\widehat{\chi}_{n}^{1 / 2} u_{n} \rightarrow \widehat{\chi}^{1 / 2} u \quad \text { in } L^{2}\left(0, T ; H_{c}\right)
$$


so that by lower semicontinuity of norm

$$
\liminf _{n \rightarrow+\infty} \int_{0}^{t} \int_{\Gamma_{c}} \widehat{\chi}_{n}\left|u_{n}\right|^{2} \geq \int_{0}^{t} \int_{\Gamma_{c}} \widehat{\chi}|u|^{2}
$$

Analogously, we can deduce that

$$
\liminf _{n \rightarrow+\infty} \int_{0}^{t} \int_{\Omega} \widehat{\beta}_{n}\left|\nabla u_{n}\right|^{2} \geq \int_{0}^{t} \int_{\Omega} \widehat{\beta}|\nabla u|^{2} .
$$

Now, combining (3.60) with (3.56) yields

$$
\limsup _{n \rightarrow+\infty} \int_{0}^{t} W^{\prime}\left\langle E_{n}, u_{n}\right\rangle_{W} \leq \int_{0}^{t} W^{\prime}\langle E, u\rangle_{W},
$$

from which we deduce $E=\mathcal{A} u$ due to [1], Lemma 1.3. In particular, it follows

$$
\lim _{n \rightarrow+\infty} \int_{0}^{t} W^{\prime}\left\langle E_{n}, u_{n}\right\rangle_{W}=\lim _{n \rightarrow+\infty} \int_{0}^{t} \int_{\Omega}\left|\nabla u_{n}\right|^{4}=\int_{0}^{t} \int_{\Omega}|\nabla u|^{4},
$$

yielding that $u_{n}$ converges strongly (see (3.36))

$$
u_{n} \rightarrow u \quad \text { in } L^{4}(0, T ; W)
$$

Then, we can identify $\zeta \in \alpha(u)$ proceeding as in (3.60) applying [1], as it holds

$$
\limsup _{n \rightarrow+\infty} \int_{0}^{t} W^{\prime}\left\langle\zeta_{n}, u_{n}\right\rangle_{W} \leq-\int_{0}^{t} \int_{\Omega}|\nabla u|^{4}-\int_{0}^{t} \int_{\Gamma_{c}} \widehat{\chi}|u|^{2}-\int_{0}^{t} \int_{\Omega} \widehat{\beta}|\nabla u|^{2}+\int_{0}^{t} W^{\prime}\langle F, u\rangle_{W}=\int_{0}^{t} W^{\prime}\langle\zeta, u\rangle_{W} .
$$

Now, we pass to the limit weakly in (3.1), written for the index $n$, as $n \rightarrow+\infty$. Owing to (3.30), (3.37), (3.40), (3.42), and (3.49), (3.64) we get at the limit (3.1). It remains to identify $\xi \in \gamma(\beta)$. We proceed as above (cf., e.g., (3.60)) by semicontinuity and test (3.1), written for $n$, by $\beta_{n}$ and integrate over $(0, t)$. Integrating by parts in time and exploiting once more $(3.30),(3.37),(3.42)$, and $(3.64)$, by lower semicontinuity of norms, we have

$$
\begin{aligned}
& \limsup _{n \rightarrow+\infty} \int_{0}^{t} V^{\prime}\left\langle\xi_{n}, \beta_{n}\right\rangle_{V}=\limsup _{n \rightarrow+\infty}-\frac{1}{2}\left\|\beta_{n}(t)\right\|_{H}^{2}+\frac{1}{2}\left\|\beta_{0}\right\|_{H}^{2}-\frac{\kappa}{2}\left\|\nabla \beta_{n}(t)\right\|_{H}^{2}+\frac{\kappa}{2}\|\nabla \beta(0)\|_{H}^{2}-\int_{0}^{t} \int_{\Omega}\left|\nabla \beta_{n}\right|^{2} \\
& -\int_{0}^{t} \int_{\Gamma_{c}}\left|\beta_{\Gamma_{\Gamma_{n}}}\right|^{2}+\int_{0}^{t} \int_{\Omega} w \beta_{n}-\frac{1}{2} \int_{0}^{t} \int_{\Omega}\left|\nabla u_{n}\right|^{2} \beta_{n} \leq-\frac{1}{2}\|\beta(t)\|_{H}^{2}+\frac{1}{2}\left\|\beta_{0}\right\|_{H}^{2}-\frac{\kappa}{2}\|\nabla \beta(t)\|_{H}^{2}+\frac{\kappa}{2}\|\nabla \beta(0)\|_{H}^{2} \\
& -\int_{0}^{t} \int_{\Omega}|\nabla \beta|^{2}-\int_{0}^{t} \int_{\Gamma_{c}}\left|\beta_{\left.\right|_{\Gamma_{c}}}\right|^{2}+\int_{0}^{t} \int_{\Omega} w \beta-\frac{1}{2} \int_{0}^{t} \int_{\Omega}|\nabla u|^{2} \beta=\int_{0}^{t} V^{\prime}\langle\xi, \beta\rangle_{V}
\end{aligned}
$$

leading to $\xi \in \gamma(\beta)$ in $V^{\prime}$ for a.e. $t$.

Finally, it is now a standard matter to pass to the limit in (3.21) exploiting (3.38), (3.50), (3.41), (3.42), and (3.64) (from which we deduce a strong convergence for the traces e.g. in $L^{4}\left(\Gamma_{c} \times(0, T)\right)$ ). Note in particular that we can directly identify $\delta \in \partial I_{[0,1]}(\chi)$ due to (3.44) and (3.50).

Eventually, the operator $\mathcal{T}$ turns out to be continuous in $\mathcal{X}$ (see (3.42) and (3.44)). By the Schauder theorem there exists a fixed point $(\beta, \chi)$ from which we have a solution to problem $(2.6),(3.1)$, and (2.8) given by $\left(\mathcal{T}_{1}(\beta, \chi), \beta, \chi\right)$ 
Remark 3.6. Let us point out that we can identify $\zeta$ and $\xi$ only in a weak sense, so that we cannot think to $\zeta$ and $\xi$ as interior constraints a.e. defined as we can do for $\delta$ in (3.21). However, our result is still physically consistent as it sufficient to deduce that $u \in D(\alpha)$ and $\beta \in D(\gamma)$, i.e., for a.e.t, $u_{\left.\right|_{\Gamma_{c}}} \leq 0$ a.e. on $\Gamma_{c}$ and $\beta \in[0,1]$ a.e. in $\Omega$.

\subsection{Passage to the limit as $\kappa \searrow 0$}

Now, we pass to the limit in (2.6), (3.1), (2.8) as $\kappa \searrow 0$ to conclude the proof of Theorem 2.1. For the sake of simplicity, for the moment we do not specify the dependence of the solution $(u, \beta, \chi)$ on $\kappa$. Then, we introduce $u_{0} \in D(\alpha)$ as the solution of (3.4) written for $t=0$ (see Lem. 3.2). We proceed formally testing (2.6) by $u_{t}$, (3.1) by $\beta_{t}$, and (2.8) by $\chi_{t}$ and adding the resulting equations. After integrating by parts in time some terms cancel. Then, using the chain rule (also for the subdifferentials) and applying the Young inequality we infer that

$$
\begin{aligned}
& \frac{1}{4}\|\nabla u(t)\|_{L^{4}(\Omega)}^{4}+\frac{1}{2} \int_{\Omega} \beta(t)|\nabla u(t)|^{2}+\frac{1}{2} \int_{\Gamma_{c}} \chi(t)\left|u_{\Gamma_{c}}(t)\right|^{2}+\left\|\beta_{t}\right\|_{L^{2}(0, t ; H)}^{2}+\kappa\left\|\nabla \beta_{t}\right\|_{L^{2}(0, t ; H)}^{2} \\
& \quad+\frac{1}{2}\|\nabla \beta(t)\|_{H}^{2}+\left\|\chi_{t}\right\|_{L^{2}\left(0, t ; H_{c}\right)}^{2}+\frac{1}{2}\|\nabla \chi(t)\|_{H}^{2}+\frac{\nu}{2}\left\|\left(\beta_{\Gamma_{\Gamma_{c}}}-\chi\right)(t)\right\|_{H_{c}}^{2} \\
& \leq \frac{1}{4}\left\|\nabla u_{0}\right\|_{L^{4}(\Omega)}^{4}+\frac{1}{2} \int_{\Omega} \beta_{0}\left|\nabla u_{0}\right|^{2}+\frac{1}{2} \int_{\Gamma_{c}} \chi_{0}\left|u_{\Gamma_{c}}(0)\right|^{2}+\frac{1}{2}\left\|\nabla \beta_{0}\right\|_{H}^{2} \\
&+\frac{1}{2}\left\|\nabla \chi_{0}\right\|_{H_{c}}^{2}+\nu\left\|\beta_{\Gamma_{c}}(0)-\chi_{0}\right\|_{H_{c}}^{2}+\left.\right|_{W^{\prime}}\langle F(t), u(t)\rangle_{W}|+| W_{W^{\prime}}\left\langle F(0), u_{0}\right\rangle_{W} \mid \\
&+\int_{0}^{t}\left|W_{W^{\prime}}\left\langle F_{t}, u\right\rangle_{W}\right|+\int_{0}^{t} \int_{\Omega}\left|w \beta_{t}\right|+\int_{0}^{t} \int_{\Gamma_{c}}\left|w_{c} \chi_{t}\right| \\
& \leq c\left(+\|F\|_{L^{\infty}\left(0, T ; W^{\prime}\right)}^{2}\right)+\int_{0}^{t}\left\|F_{t}\right\|_{W^{\prime}}\|u\|_{W}+\frac{1}{2}\left\|\beta_{t}\right\|_{L^{2}(0, t ; H)}^{2}+\frac{1}{2}\left\|\chi_{t}\right\|_{L^{2}\left(0, t ; H_{c}\right)}^{2} .
\end{aligned}
$$

Eventually, owing to (2.1) and exploiting the Gronwall lemma, we deduce the following bounds independent of $\kappa$

$$
\begin{aligned}
& \left\|u_{\kappa}\right\|_{L^{\infty}(0, T ; W)} \leq c \\
& \left\|\beta_{\kappa}\right\|_{H^{1}(0, T ; H) \cap L^{\infty}(0, T ; V)} \leq c \\
& \left\|\chi_{\kappa}\right\|_{H^{1}\left(0, T ; H_{c}\right) \cap L^{\infty}\left(0, T ; V_{c}\right)} \leq c \\
& \left\|\beta_{\kappa}^{1 / 2} \nabla u_{\kappa}\right\|_{L^{\infty}(0, T ; H)} \leq c \\
& \left\|\chi_{\kappa}^{1 / 2} u_{\left.\right|_{\Gamma_{c}, \kappa}}\right\|_{L^{\infty}\left(0, T ; H_{c}\right)} \leq c .
\end{aligned}
$$

Moreover,

$$
\kappa^{1 / 2}\left\|\nabla \partial_{t} \beta_{\kappa}\right\|_{L^{2}(0, T ; H)} \leq c .
$$

We can also test (2.8) by $-\Delta \chi_{\kappa}$, integrate over $(0, t)$ and get $(c f$. Lem. 3.4 and (3.26))

$$
\left\|\chi_{\kappa}\right\|_{L^{2}\left(0, t ; H^{2}\left(\Gamma_{c}\right)\right)} \leq c .
$$

It is now a standard matter to deduce the following weak and weak star convergences for

$$
\begin{array}{ll}
u_{\kappa} \stackrel{*}{\rightarrow} u \quad \text { in } L^{\infty}(0, T ; W), \quad u_{\kappa} \rightarrow u \quad \text { in } L^{4}(0, T ; W) \\
\beta_{\kappa} \stackrel{*}{\rightarrow} \beta \quad \text { in } H^{1}(0, T ; H) \cap L^{\infty}(0, T ; V) \\
\chi_{\kappa} \stackrel{*}{\rightarrow} \chi \quad \text { in } H^{1}\left(0, T ; H_{c}\right) \cap L^{\infty}\left(0, T ; V_{c}\right) \cap L^{2}\left(0, T ; H^{2}\left(\Gamma_{c}\right)\right) .
\end{array}
$$


Moreover,

$$
\kappa \nabla \partial_{t} \beta_{\kappa} \rightarrow 0 \quad \text { in } L^{2}(0, T ; H) .
$$

Hence, by a comparison in the equations, we obtain the analogous of (3.45)-(3.47), now written for $\zeta_{\kappa}, \xi_{\kappa}, \delta_{\kappa}$. As a consequence

$$
\begin{aligned}
& \zeta_{\kappa} \stackrel{*}{\rightarrow} \quad \text { in } L^{\infty}\left(0, T ; W^{\prime}\right), \quad \zeta_{\kappa} \rightarrow \zeta \text { in } L^{2}\left(0, T ; W^{\prime}\right), \\
& \xi_{\kappa} \rightarrow \xi \quad \text { in } L^{2}\left(0, T ; V^{\prime}\right), \\
& \delta_{\kappa} \rightarrow \delta \quad \text { in } L^{2}\left(0, T ; H_{c}\right) .
\end{aligned}
$$

We are now in the position of applying the same passage to the limit procedure as that exploited in Section 3.2 to pass to the limit as $\kappa \rightarrow 0$. Indeed, we are able to deduce exactly the same convergences as (3.40)-(3.44), (3.51), (3.54)-(3.55) (for $\chi_{\kappa}$ and $\beta_{\kappa}$ ) as they follow from (3.75)-(3.77) arguing as in Section 3.2 (cf. $(3.33),(3.35),(3.64)$, and $(3.39))$. At the limit $(u, \beta, \chi)$ turn out to solve Problem $P_{v}$.

\section{Asymptotic ANALYSIS}

In this section, we should consider the asymptotic behaviour of a solution to Problem $P_{v}$ as the interfacial coefficient $\nu \rightarrow+\infty$. This corresponds to investigate the properties of the adhesion once the interaction energy blows up.

We first perform some a priori estimates for the solution, which are independent of the interface parameter $\nu$. To this aim we have to improve the regularity required on the initial data. Indeed, let

$$
\beta_{0} \in H^{3 / 2}(\Omega), \quad \beta_{0} \in[0,1] \text { a.e. in } \Omega
$$

and fix $\chi_{0}=\beta_{0,\left.\right|_{c}}$, so that $(c f .(2.5))$

$$
\chi_{0} \in V_{c}, \quad \chi_{0} \in[0,1] \text { a.e. in } \Gamma_{c} .
$$

Then, let $\beta_{0, \nu}$ and $\chi_{0, \nu}$ satisfying (4.1), (4.2),

$$
\begin{aligned}
& \text { converging to } \beta_{0} \text { and } \chi_{0} \text { in } V \text { and } V_{c} \text { respectively, with } \\
& \nu^{1 / 2}\left\|\left.\beta_{0, \nu}\right|_{\Gamma_{c}}-\chi_{0, \nu}\right\|_{H_{c}} \leq c
\end{aligned}
$$

where $c$ does not depend on $\nu$. Then, letting $u_{0, \nu}$ be the unique solution of (3.4) written for $t=0$ where $\beta_{0, \nu}$ and $\chi_{0, \nu}$ are fixed, it follows $(c f .(3.8))$

$$
\left\|u_{0, \nu}\right\|_{W} \leq c
$$

The following lemma holds.

Lemma 4.1. Let (2.4), (4.1)-(4.3) hold (cf. also (4.4)). Denote by $\left(u_{\nu}, \beta_{\nu}, \chi_{\nu}\right)$ a solution to Problem P for $\nu>0$ fixed. Then,

$$
\begin{aligned}
& \left\|u_{\nu}\right\|_{L^{\infty}(0, T ; W)} \leq c \\
& \left\|\beta_{\nu}\right\|_{H^{1}(0, T ; H) \cap L^{\infty}(0, T ; V)} \leq c \\
& \left\|\chi_{\nu}\right\|_{H^{1}\left(0, T ; H_{c}\right) \cap L^{\infty}\left(0, T ; V_{c}\right) \cap L^{2}\left(0, T ; H^{2}\left(\Gamma_{c}\right)\right)} \leq c
\end{aligned}
$$

independently of $\nu$. 
For the sake of simplicity, for the moment we do not specify the dependence of the solution $(u, \beta, \chi)$ on $\nu$. To prove Lemma 4.1 we proceed formally and test $(2.6)$ by $u_{t},(2.7)$ by $\beta_{t}$, and $(2.8)$ by $\chi_{t}$. Then, we add the resulting equations and integrate over $(0, t)$. We get the analogous of estimates $(3.67)$, where now $\kappa=0$,

$$
\begin{aligned}
& \frac{1}{4}\|\nabla u(t)\|_{L^{4}(\Omega)}^{4}+\frac{1}{2} \int_{\Omega} \beta(t)|\nabla u(t)|^{2}+\frac{1}{2} \int_{\Gamma_{c}} \chi(t)\left|u_{\Gamma_{\Gamma_{c}}}(t)\right|^{2}+\left\|\beta_{t}\right\|_{L^{2}(0, t ; H)}^{2} \\
& \quad+\frac{1}{2}\|\nabla \beta(t)\|_{H}^{2}+\left\|\chi_{t}\right\|_{L^{2}\left(0, t ; H_{c}\right)}^{2}+\frac{1}{2}\|\nabla \chi(t)\|_{H}^{2}+\frac{\nu}{2}\left\|\left(\beta_{\Gamma_{\Gamma_{c}}}-\chi\right)(t)\right\|_{H_{c}}^{2} \\
& \leq \frac{1}{4}\left\|\nabla u_{0}\right\|_{L^{4}(\Omega)}^{4}+\frac{1}{2} \int_{\Omega} \beta_{0}\left|\nabla u_{0}\right|^{2}+\frac{1}{2} \int_{\Gamma_{c}} \chi_{0}\left|u_{\left.\right|_{\Gamma_{c}}}(0)\right|^{2}+\frac{1}{2}\left\|\nabla \beta_{0}\right\|_{H}^{2}+\frac{1}{2}\left\|\nabla \chi_{0}\right\|_{H_{c}}^{2} \\
& \quad+\nu\left\|\beta_{\Gamma_{\Gamma_{c}}}(0)-\chi_{0}\right\|_{H_{c}}^{2}+\left|W^{\prime}\langle F(t), u(t)\rangle_{W}\right|+\left|W^{\prime}\left\langle F(0), u_{0}\right\rangle_{W}\right|+\int_{0}^{t}\left|W^{\prime}\left\langle F_{t}, u\right\rangle_{W}\right| \\
& \quad+\int_{0}^{t} \int_{\Omega}\left|w \beta_{t}\right|+\int_{0}^{t} \int_{\Gamma_{c}}\left|w_{c} \chi_{t}\right| \\
& \leq c\left(1+\|F\|_{L^{\infty}\left(0, T ; W^{\prime}\right)}^{2}\right)+\int_{0}^{t}\left\|F_{t}\right\|_{W^{\prime}}\|u\|_{W}+\frac{1}{2}\left\|\beta_{t}\right\|_{L^{2}(0, t ; H)}^{2}+\frac{1}{2}\left\|\chi_{t}\right\|_{L^{2}\left(0, t ; H_{c}\right)}^{2} .
\end{aligned}
$$

Eventually, owing to (4.1)-(4.4), (2.1), and exploiting the Gronwall lemma, we deduce the following bounds independent of $\nu$ :

$$
\begin{aligned}
& \|u\|_{L^{\infty}(0, T ; W)} \leq c \\
& \|\beta\|_{H^{1}(0, T ; H) \cap L^{\infty}(0, T ; V)} \leq c \\
& \|\chi\|_{H^{1}\left(0, T ; H_{c}\right) \cap L^{\infty}\left(0, T ; V_{c}\right)} \leq c \\
& \left\|\beta^{1 / 2} \nabla u\right\|_{L^{\infty}(0, T ; H)} \leq c \\
& \left\|\chi^{1 / 2} u_{\Gamma_{\Gamma_{c}}}\right\|_{L^{\infty}\left(0, T ; H_{c}\right)} \leq c \\
& \nu^{1 / 2}\left\|\beta_{\Gamma_{\Gamma_{c}}}-\chi\right\|_{L^{\infty}\left(0, T ; H_{c}\right)} \leq c .
\end{aligned}
$$

Now, we aim to pass to the limit in (2.6)-(2.8) as $\nu \rightarrow+\infty$ and prove that at the limit we find a system solved by $(u, \beta, \chi)$. Actually, as we will show in a moment, we are able to prove that $(u, \beta, \chi)$ solve only a weaker version of our system, we are going to introduce.

By definition of $\gamma$ and $\partial I_{[0,1]}$ we can equivalently rewrite (3.1) and (2.8) as follows

$$
\begin{aligned}
& \int_{0}^{t} \int_{\Omega} \beta_{t}(\beta-\phi)+\int_{0}^{t} \int_{\Omega} \nabla \beta \cdot \nabla(\beta-\phi)+\nu \int_{0}^{t} \int_{\Gamma_{c}}\left(\beta_{\left.\right|_{\Gamma_{c}}}-\chi\right)\left(\beta_{\left.\right|_{\Gamma_{c}}}-\phi_{\left.\right|_{\Gamma_{c}}}\right) \\
& -\int_{0}^{t} \int_{\Omega} w(\beta-\phi)+\frac{1}{2} \int_{0}^{t} \int_{\Omega}|\nabla u|^{2}(\beta-\phi) \leq 0 \quad \forall \phi \in L^{2}\left(0, T ;[0,1]_{V}\right), \\
& \int_{0}^{t} \int_{\Gamma_{c}} \chi_{t}(\chi-\psi)+\int_{0}^{t} \int_{\Gamma_{c}} \nabla \chi \cdot \nabla(\chi-\psi)-\int_{0}^{t} \int_{\Gamma_{c}} w_{c}(\chi-\psi) \\
& +\frac{1}{2} \int_{0}^{t} \int_{\Gamma_{c}}\left|u_{\left.\right|_{\Gamma_{c}}}\right|^{2}(\chi-\psi)+\nu \int_{0}^{t} \int_{\Gamma_{c}}\left(\chi-\beta_{\left.\right|_{\Gamma_{c}}}\right)(\chi-\psi) \quad \forall \psi \in L^{2}\left(0, T ; V_{c}\right), \\
& \psi \in[0,1] \text { a.e. in } \Gamma_{c} \times(0, T) .
\end{aligned}
$$

Then, to pass to the limit in (4.15)-(4.16), we restrict our analysis to consider as test functions in (4.15)

$$
\phi \in L^{2}\left(0, T ; H^{3 / 2}(\Omega)\right) \text { s.t. } \phi \in[0,1] \text { a.e. in } \Omega \times(0, T),
$$


so that $\phi_{\left.\right|_{\Gamma_{c}}} \in L^{2}\left(0, T ; V_{c}\right)$ and $\phi_{\left.\right|_{\Gamma_{c}}} \in[0,1]$ a.e. in $\Gamma_{c} \times(0, T)$. In particular, we are allowed to take as test function in (4.16) $\psi=\phi_{\Gamma_{\Gamma_{c}}}$. Then, adding the resulting inequalities we get

$$
\begin{aligned}
& \int_{0}^{t} \int_{\Omega} \beta_{t}(\beta-\phi)+\int_{0}^{t} \int_{\Omega} \nabla \beta \cdot \nabla(\beta-\phi)+\nu \int_{0}^{t} \int_{\Gamma_{c}}\left(\beta_{\Gamma_{\Gamma_{c}}}-\chi\right)^{2}-\int_{0}^{t} \int_{\Omega} w(\beta-\phi) \\
& +\frac{1}{2} \int_{0}^{t} \int_{\Omega}|\nabla u|^{2}(\beta-\phi)+\int_{0}^{t} \int_{\Gamma_{c}} \chi_{t}\left(\chi-\phi_{\Gamma_{\Gamma_{c}}}\right)+\int_{0}^{t} \int_{\Gamma_{c}} \nabla \chi \cdot \nabla\left(\chi-\phi_{\left.\right|_{\Gamma_{c}}}\right) \\
& -\int_{0}^{t} \int_{\Gamma_{c}} w_{c}\left(\chi-\phi_{\left.\right|_{\Gamma_{c}}}\right)+\frac{1}{2} \int_{0}^{t} \int_{\Gamma_{c}}\left|u_{\left.\right|_{\Gamma_{c}}}\right|^{2}\left(\chi-\phi_{\left.\right|_{\Gamma_{c}}}\right) \leq 0
\end{aligned}
$$

and also, as $\nu \int_{\Gamma_{c}}\left(\beta_{\Gamma_{\Gamma_{c}}}-\chi\right)^{2} \geq 0$,

$$
\begin{aligned}
& \int_{0}^{t} \int_{\Omega} \beta_{t}(\beta-\phi)+\int_{0}^{t} \int_{\Omega} \nabla \beta \cdot \nabla(\beta-\phi)-\int_{0}^{t} \int_{\Omega} w(\beta-\phi) \\
& +\frac{1}{2} \int_{0}^{t} \int_{\Omega}|\nabla u|^{2}(\beta-\phi)+\int_{0}^{t} \int_{\Gamma_{c}} \chi_{t}\left(\chi-\phi_{\mid \Gamma_{c}}\right)+\int_{0}^{t} \int_{\Gamma_{c}} \nabla \chi \cdot \nabla\left(\chi-\phi_{\left.\right|_{\Gamma_{c}}}\right) \\
& -\int_{0}^{t} \int_{\Gamma_{c}} w_{c}\left(\chi-\phi_{\Gamma_{\Gamma_{c}}}\right)+\frac{1}{2} \int_{0}^{t} \int_{\Gamma_{c}}\left|u_{\left.\right|_{\Gamma_{c}}}\right|^{2}\left(\chi-\phi_{\left.\right|_{\Gamma_{c}}}\right) \leq 0
\end{aligned}
$$

for all $\phi \in L^{2}\left(0, T ; H^{3 / 2}(\Omega)\right)$ s.t. $\phi \in[0,1]$ a.e. in $\Omega \times(0, T)$.

The following theorem holds.

Theorem 4.1. Let (2.4), (4.1)-(4.4) hold. Then, as $\nu \rightarrow+\infty$, at least for some subsequence

$$
\begin{array}{ll}
u_{\nu} \stackrel{*}{\rightarrow} u & \text { in } L^{\infty}(0, T ; W) \\
\beta_{\nu} \stackrel{*}{\rightarrow} \beta & \text { in } H^{1}(0, T ; H) \cap L^{\infty}(0, T ; V) \\
\chi_{\nu} \stackrel{*}{\rightarrow} \chi \quad \text { in } H^{1}\left(0, T ; H_{c}\right) \cap L^{\infty}\left(0, T ; V_{c}\right) .
\end{array}
$$

Moreover $\beta_{\left.\right|_{\Gamma_{c}}}=\chi$ a.e. and $(u, \beta, \chi)$ solve for a.e. $t \in(0, T)(3.4)$ and $(4.18)$ for any $\phi \in L^{2}\left(0, T ; H^{3 / 2}(\Omega)\right)$ s.t. $\phi \in[0,1]$ a.e. in $\Omega \times(0, T)$.

Convergences (4.19)-(4.21) easily follows by Lemma 4.1. Hence, (4.20) and (4.21) lead to

$$
\begin{array}{ll}
\beta_{\nu} \rightarrow \beta & \text { in } C^{0}\left([0, T] ; H^{1-\varepsilon}(\Omega)\right), \\
\chi_{\nu} \rightarrow \chi & \text { in } C^{0}\left([0, T] ; H^{1-\varepsilon}\left(\Gamma_{c}\right)\right) .
\end{array}
$$

In particular, (4.22) implies

$$
\beta_{\nu} \rightarrow \beta_{\Gamma_{\Gamma_{c}}} \text { in } C^{0}\left([0, T] ; L^{p}\left(\Gamma_{c}\right)\right), p<4 .
$$

Moreover, (4.14) and (4.22)-(4.24) imply

$$
\beta_{\nu}-\chi_{\nu} \rightarrow 0 \text { in } L^{\infty}\left(0, T ; H_{c}\right)
$$

and

$$
\beta_{\left.\right|_{\Gamma_{c}}}=\chi \quad \text { a.e. in } \Gamma_{c} \times(0, T) .
$$

Now, we pass to the limit in (2.6) and (4.18). We first proceed investigating the asymptotic behaviour of the equation (2.6). Due to Lemma 4.1, we are allowed apply the same arguments we have exploited in Section 3 
to pass to the limit in (2.6) (written for $n$ as $n \rightarrow+\infty$ ). In particular, let us recall that we get, e.g.,

$$
u_{\nu} \rightarrow u \quad \text { in } L^{4}(0, T ; W) .
$$

Hence, we pass to the limit in (4.18) written for $\nu$. By lower semicontinuity of norms, weak(weak star)-strong convergence, (4.19)-(4.24), (4.27), and (4.26) it is now a standard matter to infer that

$$
\begin{aligned}
& \int_{0}^{t} \int_{\Omega} \beta_{t} \beta+\int_{0}^{t} \int_{\Omega}|\nabla \beta|^{2}-\int_{0}^{t} \int_{\Omega} w \beta+\frac{1}{2} \int_{0}^{t} \int_{\Omega}|\nabla u|^{2} \beta \\
& +\int_{0}^{t} \int_{\Gamma_{c}} \partial_{t} \beta_{\left.\right|_{\Gamma_{c}}} \beta_{\left.\right|_{\Gamma_{c}}}+\int_{0}^{t} \int_{\Gamma_{c}}\left|\nabla \beta_{\Gamma_{\Gamma_{c}}}\right|^{2}-\int_{0}^{t} \int_{\Gamma_{c}} w_{c} \beta_{\Gamma_{\Gamma_{c}}}+\frac{1}{2} \int_{0}^{t} \int_{\Gamma_{c}}|u|^{2} \beta_{\Gamma_{\Gamma_{c}}} \\
& \leq \liminf _{\nu \rightarrow+\infty}^{t} \int_{0}^{t} \int_{\Omega} \partial_{t} \beta_{\nu} \beta_{\nu}+\int_{0}^{t} \int_{\Omega}\left|\nabla \beta_{\nu}\right|^{2}-\int_{0}^{t} \int_{\Omega} w \beta_{\nu}+\frac{1}{2} \int_{0}^{t} \int_{\Omega}\left|\nabla u_{\nu}\right|^{2} \beta_{\nu} \\
& \quad+\int_{0}^{t} \int_{\Gamma_{c}} \partial_{t} \chi_{\nu} \chi_{\nu}+\int_{0}^{t} \int_{\Gamma_{c}}\left|\nabla \chi_{\nu}\right|^{2}-\int_{0}^{t} \int_{\Gamma_{c}} w_{c} \chi_{\nu}+\frac{1}{2} \int_{0}^{t} \int_{\Gamma_{c}}\left|u_{\nu}\right|^{2} \chi_{\nu} \\
& \leq-\int_{0}^{t} \int_{\Omega} \beta_{t} \phi-\int_{0}^{t} \int_{\Omega} \nabla \beta \cdot \nabla \phi+\int_{0}^{t} \int_{\Omega} w \phi-\frac{1}{2} \int_{0}^{t} \int_{\Omega}|\nabla u|^{2} \phi \\
& \quad-\int_{0}^{t} \int_{\Gamma_{c}} \partial_{t} \beta_{\Gamma_{\Gamma_{c}}} \phi_{\left.\right|_{\Gamma_{c}}}-\int_{0}^{t} \int_{\Gamma_{c}} \nabla \beta_{\Gamma_{\Gamma_{c}}} \cdot \nabla \phi_{\left.\right|_{\Gamma_{c}}}+\int_{0}^{t} \int_{\Gamma_{c}} w_{c} \phi_{\left.\right|_{\Gamma_{c}}}-\frac{1}{2} \int_{0}^{t} \int_{\Gamma_{c}}|u|^{2} \phi_{\left.\right|_{\Gamma_{c}}}
\end{aligned}
$$

which is our expected result.

Remark 4.2. Let us point out that (4.28) yields (for $\beta$ sufficiently regular) a variational inclusion with a subdifferential. Indeed, let $\pi \in \partial_{H^{3 / 2}(\Omega),\left(H^{3 / 2}(\Omega)\right)^{\prime}} I_{[0,1]_{H^{3 / 2}}}(\beta)$ for a.e. $t$, where

$$
[0,1]_{H^{3 / 2}}=\left\{\phi \in H^{3 / 2}(\Omega) \text { s.t. } \phi \in[0,1] \text { a.e. in } \Omega\right\} \text {. }
$$

Thus, (4.28) leads to

$$
\begin{aligned}
& \int_{0}^{t} \int_{\Omega} \beta_{t} \phi+\int_{0}^{t} \int_{\Omega} \nabla \beta \cdot \nabla \phi+\int_{0}^{t} \int_{\Gamma_{c}} \partial_{t} \beta_{\left.\right|_{\Gamma_{c}}} \phi_{\left.\right|_{\Gamma_{c}}}+\int_{0}^{t} \int_{\Gamma_{c}} \nabla \beta_{\left.\right|_{\Gamma_{c}}} \cdot \nabla \phi_{\left.\right|_{\Gamma_{c}}}+\int_{0}^{t}\left(H^{3 / 2}(\Omega)\right)^{\prime}\langle\pi, \phi\rangle_{H^{3 / 2}(\Omega)} \\
& =\int_{0}^{t} \int_{\Omega} w \phi+\frac{1}{2} \int_{0}^{t} \int_{\Omega}|\nabla u|^{2} \phi+\int_{0}^{t} \int_{\Gamma_{c}} w_{c} \phi_{\left.\right|_{\Gamma_{c}}}+\frac{1}{2} \int_{0}^{t} \int_{\Gamma_{c}}\left|u_{\left.\right|_{\Gamma_{c}}}\right|^{2} \phi_{\left.\right|_{\Gamma_{c}}},
\end{aligned}
$$

for any $\phi \in L^{2}\left(0, T ;[0,1]_{H^{3 / 2}}\right)$, which can be read as a variational inclusion with "dynamic boundary conditions".

\section{REFERENCES}

[1] V. Barbu, Nonlinear Semigroups and Differential Equations in Banach Spaces. Noordhoff, Leyden (1976).

[2] E. Bonetti and G. Bonfanti, Well-posedness results for a model of damage in thermoviscoelastic materials. Ann. Inst. H. Poincaré Anal. Non Linéaire 6 (2008) 1187-1208.

[3] E. Bonetti and M. Frémond, Collisions and fracture, a 1-D example: How to tear off a chandelier from the ceiling. J. Elast. 74 (2004) 47-66.

[4] E. Bonetti and G. Schimperna, Local existence for Frémond's model of damage in elastic materials. Contin. Mech. Thermodyn. 16 (2004) 319-335.

[5] E. Bonetti, A. Segatti and G. Schimperna, On a doubly nonlinear model for the evolution of damaging in viscoelastic materials. J. Diff. Equ. 218 (2005) 91-116.

[6] E. Bonetti, G. Bonfanti and R. Rossi, Well-posedness and long-time behaviour for a model of contact with adhesion. Indiana Univ. Math. J. 56 (2007) 2787-2819.

[7] E. Bonetti, G. Bonfanti and R. Rossi, Global existence for a contact problem with adhesion. Math. Meth. Appl. Sci. 31 (2008) 1029-1064. 
[8] E. Bonetti, G. Bonfanti and R. Rossi, Thermal effects in adhesive contact: modelling and analysis. Nonlinearity 22 (2009) $2697-2731$.

[9] P. Colli, F. Luterotti, G. Schimperna and U. Stefanelli, Global existence for a class of generalized systems for irreversible phase changes. NoDEA Nonlinear Diff. Equ. Appl. 9 (2002) 255-276.

[10] F. Freddi and M. Frémond, Damage in domains and interfaces: a coupled predictive theory. J. Mech. Mater. Struct. 7 (2006) $1205-1233$.

[11] M. Frémond, Équilibre des structures qui adhèrent à leur support. C. R. Acad. Sci. Paris 295 (1982) 913-916.

[12] M. Frémond, Adhérence des solides. J. Méc. Théor. Appl. 6 (1987) 383-407.

[13] M. Frémond, Non-smooth Thermomechanics. Springer-Verlag, Berlin (2002).

[14] M. Frémond, Collisions. Edizioni del Dipartimento di Ingegneria Civile dell' Università di Roma Tor Vergata, Italy (2007).

[15] M. Frémond and N. Kenmochi, Damage problems for viscous locking materials. Adv. Math. Sci. Appl. 16 (2006) $697-716$.

[16] M. Frémond and B. Nedjar, Damage, gradient of damage and priciple of virtual power. Int. J. Solids Struct. 33 (1996) $1083-1103$

[17] M. Frémond, K. Kuttler and M. Shillor, Existence and uniqueness of solutions for a dynamic one-dimensional damage model. J. Math. Anal. Appl. 229 (1999) 271-294.

[18] J.L. Lions, Quelques méthodes de résolution des problèmes aux limites non linéaires. Dunod Gauthier-Villars, Paris (1969).

[19] J.J. Moreau, Sur les lois de frottement, de viscosité et plasticité. C. R. Acad. Sci. Paris Sér. II Méc. Phys. Chim. Sci. Univers Sci. Terre 271 (1970) 608-611.

[20] N. Point, Unilateral contact with adherence. Math. Meth. Appl. Sci. 10 (1998) 367-381.

[21] J. Simon, Compact sets in the space $L^{p}(0, T ; B)$. Ann. Mat. Pura Appl. 146 (1987) 65-96. 